\title{
Artificial Neural Network Prediction of Metastable Zone Widths in Reactive Crystallization of Lithium Carbonate
}

Siyang Ma ${ }^{1}$, Chao $\mathrm{Li}^{2}$, Jie Gao ${ }^{2}$, He Yang ${ }^{1}$, Weiwei Tang ${ }^{1}$, Junbo Gong ${ }^{1}$, Zhenguo Gao ${ }^{1 *}$

1. Tianjin University, School of Chemical Engineering and Technology, State Key

Laboratory of Chemical Engineering, Tianjin, 300072, P. R. China

2. Tianqi Lithium (Jiangsu) Co., Ltd., Zhang Jiagang 215634, P. R. China

* Corresponding author: zhenguogao@tju.edu.cn

\section{Supporting Information}

Table $\mathrm{S} 1$ is the measured supersolubility of $\mathrm{Li}_{2} \mathrm{CO}_{3}$ at different process parameters.

Table $\mathrm{S} 2$ is the measured supersolubility of $\mathrm{Li}_{2} \mathrm{CO}_{3}$ based on orthogonal experimental design.

Table S1 Measured supersolubility of $\mathrm{Li}_{2} \mathrm{CO}_{3}$ at different process parameters

\begin{tabular}{ccccccccc}
\hline $\begin{array}{c}\text { Temperature } \\
(\mathrm{K})\end{array}$ & $\begin{array}{c}\text { agitation } \\
\text { speed } \\
(\mathrm{rpm})\end{array}$ & $\begin{array}{c}\text { Volume } \\
(\mathrm{L})\end{array}$ & $\begin{array}{c}\mathrm{c}\left(\mathrm{K}_{2} \mathrm{SO}_{4}\right) \\
(\mathrm{mol} / \mathrm{L})\end{array}$ & $\begin{array}{c}\mathrm{c}(\mathrm{NaCl}) \\
(\mathrm{mol} / \mathrm{L})\end{array}$ & $\begin{array}{c}\mathrm{c}\left(\mathrm{Li}_{2} \mathrm{SO}_{4}\right) \\
(\mathrm{mol} / \mathrm{L})\end{array}$ & $\begin{array}{c}\mathrm{c}\left(\mathrm{Na}_{2} \mathrm{CO}_{3}\right) \\
(\mathrm{mol} / \mathrm{L})\end{array}$ & $\begin{array}{c}\mathrm{Na}_{2} \mathrm{CO}_{3} \\
\text { feed rate } \\
(\mathrm{ml} / \mathrm{min})\end{array}$ & $\begin{array}{c}c_{S S} \\
(\mathrm{~mol} / \mathrm{L})\end{array}$ \\
\hline 313.15 & 100 & 0.1 & 0.0 & 0.0 & 1.0 & 1.0 & 2.0 & 0.33119 \\
313.15 & 100 & 0.2 & 0.0 & 0.0 & 1.0 & 1.0 & 2.0 & 0.32718 \\
313.15 & 100 & 0.5 & 0.0 & 0.0 & 1.0 & 1.0 & 2.0 & 0.32356 \\
313.15 & 100 & 1.0 & 0.0 & 0.0 & 1.0 & 1.0 & 2.0 & 0.31536 \\
313.15 & 100 & 0.5 & 0.5 & 0.0 & 1.0 & 1.0 & 2.0 & 0.32087 \\
313.15 & 100 & 0.5 & 1.0 & 0.0 & 1.0 & 1.0 & 2.0 & 0.31270 \\
313.15 & 100 & 0.5 & 1.5 & 0.0 & 1.0 & 1.0 & 2.0 & 0.30746 \\
313.15 & 100 & 0.5 & 0.0 & 0.5 & 1.0 & 1.0 & 2.0 & 0.31536 \\
313.15 & 100 & 0.5 & 0.0 & 1.0 & 1.0 & 1.0 & 2.0 & 0.31214 \\
313.15 & 100 & 0.5 & 0.0 & 1.5 & 1.0 & 1.0 & 2.0 & 0.30726 \\
313.15 & 100 & 0.5 & 0.0 & 0.0 & 1.5 & 1.0 & 2.0 & 0.31714 \\
313.15 & 100 & 0.5 & 0.0 & 0.0 & 2.0 & 1.0 & 2.0 & 0.31141 \\
313.15 & 100 & 0.5 & 0.0 & 0.0 & 2.5 & 1.0 & 2.0 & 0.30615 \\
313.15 & 100 & 0.5 & 0.0 & 0.0 & 1.0 & 1.5 & 2.0 & 0.32081 \\
313.15 & 100 & 0.5 & 0.0 & 0.0 & 1.0 & 2.0 & 2.0 & 0.31731 \\
313.15 & 100 & 0.5 & 0.0 & 0.0 & 1.0 & 2.5 & 2.0 & 0.31467 \\
313.15 & 100 & 0.5 & 0.0 & 0.0 & 1.0 & 1.0 & 2.5 & 0.31714 \\
313.15 & 100 & 0.5 & 0.0 & 0.0 & 1.0 & 1.0 & 3.0 & 0.31719 \\
313.15 & 100 & 0.5 & 0.0 & 0.0 & 1.0 & 1.0 & 3.5 & 0.31533 \\
313.15 & 100 & 0.5 & 0.0 & 0.0 & 1.0 & 1.0 & 4.0 & 0.31721 \\
313.15 & 200 & 0.1 & 0.0 & 0.0 & 1.0 & 1.0 & 2.0 & 0.32755 \\
313.15 & 200 & 0.2 & 0.0 & 0.0 & 1.0 & 1.0 & 2.0 & 0.32382
\end{tabular}




\begin{tabular}{|c|c|c|c|c|c|c|c|c|}
\hline 313.15 & 200 & 0.5 & 0.0 & 0.0 & 1.0 & 1.0 & 2.0 & 0.32019 \\
\hline 313.15 & 200 & 1.0 & 0.0 & 0.0 & 1.0 & 1.0 & 2.0 & 0.31216 \\
\hline 313.15 & 200 & 0.5 & 0.5 & 0.0 & 1.0 & 1.0 & 2.0 & 0.31752 \\
\hline 313.15 & 200 & 0.5 & 1.0 & 0.0 & 1.0 & 1.0 & 2.0 & 0.30961 \\
\hline 313.15 & 200 & 0.5 & 1.5 & 0.0 & 1.0 & 1.0 & 2.0 & 0.30431 \\
\hline 313.15 & 200 & 0.5 & 0.0 & 0.5 & 1.0 & 1.0 & 2.0 & 0.31211 \\
\hline 313.15 & 200 & 0.5 & 0.0 & 1.0 & 1.0 & 1.0 & 2.0 & 0.30890 \\
\hline 313.15 & 200 & 0.5 & 0.0 & 1.5 & 1.0 & 1.0 & 2.0 & 0.30414 \\
\hline 313.15 & 200 & 0.5 & 0.0 & 0.0 & 1.5 & 1.0 & 2.0 & 0.31389 \\
\hline 313.15 & 200 & 0.5 & 0.0 & 0.0 & 2.0 & 1.0 & 2.0 & 0.30824 \\
\hline 313.15 & 200 & 0.5 & 0.0 & 0.0 & 2.5 & 1.0 & 2.0 & 0.30304 \\
\hline 313.15 & 200 & 0.5 & 0.0 & 0.0 & 1.0 & 1.5 & 2.0 & 0.31752 \\
\hline 313.15 & 200 & 0.5 & 0.0 & 0.0 & 1.0 & 2.0 & 2.0 & 0.31409 \\
\hline 313.15 & 200 & 0.5 & 0.0 & 0.0 & 1.0 & 2.5 & 2.0 & 0.31138 \\
\hline 313.15 & 200 & 0.5 & 0.0 & 0.0 & 1.0 & 1.0 & 2.5 & 0.31394 \\
\hline 313.15 & 200 & 0.5 & 0.0 & 0.0 & 1.0 & 1.0 & 3.0 & 0.31405 \\
\hline 313.15 & 200 & 0.5 & 0.0 & 0.0 & 1.0 & 1.0 & 3.5 & 0.31209 \\
\hline 313.15 & 200 & 0.5 & 0.0 & 0.0 & 1.0 & 1.0 & 4.0 & 0.31394 \\
\hline 313.15 & 300 & 0.1 & 0.0 & 0.0 & 1.0 & 1.0 & 2.0 & 0.31607 \\
\hline 313.15 & 300 & 0.2 & 0.0 & 0.0 & 1.0 & 1.0 & 2.0 & 0.31254 \\
\hline 313.15 & 300 & 0.5 & 0.0 & 0.0 & 1.0 & 1.0 & 2.0 & 0.30915 \\
\hline 313.15 & 300 & 1.0 & 0.0 & 0.0 & 1.0 & 1.0 & 2.0 & 0.30144 \\
\hline 313.15 & 300 & 0.5 & 0.5 & 0.0 & 1.0 & 1.0 & 2.0 & 0.30652 \\
\hline 313.15 & 300 & 0.5 & 1.0 & 0.0 & 1.0 & 1.0 & 2.0 & 0.29883 \\
\hline 313.15 & 300 & 0.5 & 1.5 & 0.0 & 1.0 & 1.0 & 2.0 & 0.29387 \\
\hline 313.15 & 300 & 0.5 & 0.0 & 0.5 & 1.0 & 1.0 & 2.0 & 0.30135 \\
\hline 313.15 & 300 & 0.5 & 0.0 & 1.0 & 1.0 & 1.0 & 2.0 & 0.29834 \\
\hline 313.15 & 300 & 0.5 & 0.0 & 1.5 & 1.0 & 1.0 & 2.0 & 0.29361 \\
\hline 313.15 & 300 & 0.5 & 0.0 & 0.0 & 1.5 & 1.0 & 2.0 & 0.30309 \\
\hline 313.15 & 300 & 0.5 & 0.0 & 0.0 & 2.0 & 1.0 & 2.0 & 0.29773 \\
\hline 313.15 & 300 & 0.5 & 0.0 & 0.0 & 2.5 & 1.0 & 2.0 & 0.29262 \\
\hline 313.15 & 300 & 0.5 & 0.0 & 0.0 & 1.0 & 1.5 & 2.0 & 0.30649 \\
\hline 313.15 & 300 & 0.5 & 0.0 & 0.0 & 1.0 & 2.0 & 2.0 & 0.30312 \\
\hline 313.15 & 300 & 0.5 & 0.0 & 0.0 & 1.0 & 2.5 & 2.0 & 0.30065 \\
\hline 313.15 & 300 & 0.5 & 0.0 & 0.0 & 1.0 & 1.0 & 2.5 & 0.30303 \\
\hline 313.15 & 300 & 0.5 & 0.0 & 0.0 & 1.0 & 1.0 & 3.0 & 0.30320 \\
\hline 313.15 & 300 & 0.5 & 0.0 & 0.0 & 1.0 & 1.0 & 3.5 & 0.30131 \\
\hline 313.15 & 300 & 0.5 & 0.0 & 0.0 & 1.0 & 1.0 & 4.0 & 0.30317 \\
\hline 313.15 & 400 & 0.1 & 0.0 & 0.0 & 1.0 & 1.0 & 2.0 & 0.30988 \\
\hline 313.15 & 400 & 0.2 & 0.0 & 0.0 & 1.0 & 1.0 & 2.0 & 0.30651 \\
\hline 313.15 & 400 & 0.5 & 0.0 & 0.0 & 1.0 & 1.0 & 2.0 & 0.30311 \\
\hline 313.15 & 400 & 1.0 & 0.0 & 0.0 & 1.0 & 1.0 & 2.0 & 0.29560 \\
\hline 313.15 & 400 & 0.5 & 0.5 & 0.0 & 1.0 & 1.0 & 2.0 & 0.30067 \\
\hline 313.15 & 400 & 0.5 & 1.0 & 0.0 & 1.0 & 1.0 & 2.0 & 0.29311 \\
\hline
\end{tabular}




\begin{tabular}{|c|c|c|c|c|c|c|c|c|}
\hline 313.15 & 400 & 0.5 & 1.5 & 0.0 & 1.0 & 1.0 & 2.0 & 0.28819 \\
\hline 313.15 & 400 & 0.5 & 0.0 & 0.5 & 1.0 & 1.0 & 2.0 & 0.29563 \\
\hline 313.15 & 400 & 0.5 & 0.0 & 1.0 & 1.0 & 1.0 & 2.0 & 0.29252 \\
\hline 313.15 & 400 & 0.5 & 0.0 & 1.5 & 1.0 & 1.0 & 2.0 & 0.28803 \\
\hline 313.15 & 400 & 0.5 & 0.0 & 0.0 & 1.5 & 1.0 & 2.0 & 0.29717 \\
\hline 313.15 & 400 & 0.5 & 0.0 & 0.0 & 2.0 & 1.0 & 2.0 & 0.29194 \\
\hline 313.15 & 400 & 0.5 & 0.0 & 0.0 & 2.5 & 1.0 & 2.0 & 0.28700 \\
\hline 313.15 & 400 & 0.5 & 0.0 & 0.0 & 1.0 & 1.5 & 2.0 & 0.30059 \\
\hline 313.15 & 400 & 0.5 & 0.0 & 0.0 & 1.0 & 2.0 & 2.0 & 0.29727 \\
\hline 313.15 & 400 & 0.5 & 0.0 & 0.0 & 1.0 & 2.5 & 2.0 & 0.29486 \\
\hline 313.15 & 400 & 0.5 & 0.0 & 0.0 & 1.0 & 1.0 & 2.5 & 0.29718 \\
\hline 313.15 & 400 & 0.5 & 0.0 & 0.0 & 1.0 & 1.0 & 3.0 & 0.29735 \\
\hline 313.15 & 400 & 0.5 & 0.0 & 0.0 & 1.0 & 1.0 & 3.5 & 0.29550 \\
\hline 313.15 & 400 & 0.5 & 0.0 & 0.0 & 1.0 & 1.0 & 4.0 & 0.29724 \\
\hline 323.15 & 100 & 0.1 & 0.0 & 0.0 & 1.0 & 1.0 & 2.0 & 0.28584 \\
\hline 323.15 & 100 & 0.2 & 0.0 & 0.0 & 1.0 & 1.0 & 2.0 & 0.28280 \\
\hline 323.15 & 100 & 0.5 & 0.0 & 0.0 & 1.0 & 1.0 & 2.0 & 0.27962 \\
\hline 323.15 & 100 & 1.0 & 0.0 & 0.0 & 1.0 & 1.0 & 2.0 & 0.27265 \\
\hline 323.15 & 100 & 0.5 & 0.5 & 0.0 & 1.0 & 1.0 & 2.0 & 0.27733 \\
\hline 323.15 & 100 & 0.5 & 1.0 & 0.0 & 1.0 & 1.0 & 2.0 & 0.27042 \\
\hline 323.15 & 100 & 0.5 & 1.5 & 0.0 & 1.0 & 1.0 & 2.0 & 0.26603 \\
\hline 323.15 & 100 & 0.5 & 0.0 & 0.5 & 1.0 & 1.0 & 2.0 & 0.27270 \\
\hline 323.15 & 100 & 0.5 & 0.0 & 1.0 & 1.0 & 1.0 & 2.0 & 0.26989 \\
\hline 323.15 & 100 & 0.5 & 0.0 & 1.5 & 1.0 & 1.0 & 2.0 & 0.26573 \\
\hline 323.15 & 100 & 0.5 & 0.0 & 0.0 & 1.5 & 1.0 & 2.0 & 0.27415 \\
\hline 323.15 & 100 & 0.5 & 0.0 & 0.0 & 2.0 & 1.0 & 2.0 & 0.26937 \\
\hline 323.15 & 100 & 0.5 & 0.0 & 0.0 & 2.5 & 1.0 & 2.0 & 0.26483 \\
\hline 323.15 & 100 & 0.5 & 0.0 & 0.0 & 1.0 & 1.5 & 2.0 & 0.27739 \\
\hline 323.15 & 100 & 0.5 & 0.0 & 0.0 & 1.0 & 2.0 & 2.0 & 0.27428 \\
\hline 323.15 & 100 & 0.5 & 0.0 & 0.0 & 1.0 & 2.5 & 2.0 & 0.27199 \\
\hline 323.15 & 100 & 0.5 & 0.0 & 0.0 & 1.0 & 1.0 & 2.5 & 0.27417 \\
\hline 323.15 & 100 & 0.5 & 0.0 & 0.0 & 1.0 & 1.0 & 3.0 & 0.27422 \\
\hline 323.15 & 100 & 0.5 & 0.0 & 0.0 & 1.0 & 1.0 & 3.5 & 0.27265 \\
\hline 323.15 & 100 & 0.5 & 0.0 & 0.0 & 1.0 & 1.0 & 4.0 & 0.27424 \\
\hline 323.15 & 200 & 0.1 & 0.0 & 0.0 & 1.0 & 1.0 & 2.0 & 0.28304 \\
\hline 323.15 & 200 & 0.2 & 0.0 & 0.0 & 1.0 & 1.0 & 2.0 & 0.27988 \\
\hline 323.15 & 200 & 0.5 & 0.0 & 0.0 & 1.0 & 1.0 & 2.0 & 0.27679 \\
\hline 323.15 & 200 & 1.0 & 0.0 & 0.0 & 1.0 & 1.0 & 2.0 & 0.26994 \\
\hline 323.15 & 200 & 0.5 & 0.5 & 0.0 & 1.0 & 1.0 & 2.0 & 0.27448 \\
\hline 323.15 & 200 & 0.5 & 1.0 & 0.0 & 1.0 & 1.0 & 2.0 & 0.26774 \\
\hline 323.15 & 200 & 0.5 & 1.5 & 0.0 & 1.0 & 1.0 & 2.0 & 0.26328 \\
\hline 323.15 & 200 & 0.5 & 0.0 & 0.5 & 1.0 & 1.0 & 2.0 & 0.27002 \\
\hline 323.15 & 200 & 0.5 & 0.0 & 1.0 & 1.0 & 1.0 & 2.0 & 0.26719 \\
\hline 323.15 & 200 & 0.5 & 0.0 & 1.5 & 1.0 & 1.0 & 2.0 & 0.26304 \\
\hline
\end{tabular}




\begin{tabular}{|c|c|c|c|c|c|c|c|c|}
\hline 323.15 & 200 & 0.5 & 0.0 & 0.0 & 1.5 & 1.0 & 2.0 & 0.27142 \\
\hline 323.15 & 200 & 0.5 & 0.0 & 0.0 & 2.0 & 1.0 & 2.0 & 0.26673 \\
\hline 323.15 & 200 & 0.5 & 0.0 & 0.0 & 2.5 & 1.0 & 2.0 & 0.26219 \\
\hline 323.15 & 200 & 0.5 & 0.0 & 0.0 & 1.0 & 1.5 & 2.0 & 0.27453 \\
\hline 323.15 & 200 & 0.5 & 0.0 & 0.0 & 1.0 & 2.0 & 2.0 & 0.27152 \\
\hline 323.15 & 200 & 0.5 & 0.0 & 0.0 & 1.0 & 2.5 & 2.0 & 0.26929 \\
\hline 323.15 & 200 & 0.5 & 0.0 & 0.0 & 1.0 & 1.0 & 2.5 & 0.27140 \\
\hline 323.15 & 200 & 0.5 & 0.0 & 0.0 & 1.0 & 1.0 & 3.0 & 0.27152 \\
\hline 323.15 & 200 & 0.5 & 0.0 & 0.0 & 1.0 & 1.0 & 3.5 & 0.26989 \\
\hline 323.15 & 200 & 0.5 & 0.0 & 0.0 & 1.0 & 1.0 & 4.0 & 0.27158 \\
\hline 323.15 & 300 & 0.1 & 0.0 & 0.0 & 1.0 & 1.0 & 2.0 & 0.27323 \\
\hline 323.15 & 300 & 0.2 & 0.0 & 0.0 & 1.0 & 1.0 & 2.0 & 0.27031 \\
\hline 323.15 & 300 & 0.5 & 0.0 & 0.0 & 1.0 & 1.0 & 2.0 & 0.26735 \\
\hline 323.15 & 300 & 1.0 & 0.0 & 0.0 & 1.0 & 1.0 & 2.0 & 0.26086 \\
\hline 323.15 & 300 & 0.5 & 0.5 & 0.0 & 1.0 & 1.0 & 2.0 & 0.26517 \\
\hline 323.15 & 300 & 0.5 & 1.0 & 0.0 & 1.0 & 1.0 & 2.0 & 0.25863 \\
\hline 323.15 & 300 & 0.5 & 1.5 & 0.0 & 1.0 & 1.0 & 2.0 & 0.25440 \\
\hline 323.15 & 300 & 0.5 & 0.0 & 0.5 & 1.0 & 1.0 & 2.0 & 0.26086 \\
\hline 323.15 & 300 & 0.5 & 0.0 & 1.0 & 1.0 & 1.0 & 2.0 & 0.25815 \\
\hline 323.15 & 300 & 0.5 & 0.0 & 1.5 & 1.0 & 1.0 & 2.0 & 0.25422 \\
\hline 323.15 & 300 & 0.5 & 0.0 & 0.0 & 1.5 & 1.0 & 2.0 & 0.26220 \\
\hline 323.15 & 300 & 0.5 & 0.0 & 0.0 & 2.0 & 1.0 & 2.0 & 0.25763 \\
\hline 323.15 & 300 & 0.5 & 0.0 & 0.0 & 2.5 & 1.0 & 2.0 & 0.25335 \\
\hline 323.15 & 300 & 0.5 & 0.0 & 0.0 & 1.0 & 1.5 & 2.0 & 0.26525 \\
\hline 323.15 & 300 & 0.5 & 0.0 & 0.0 & 1.0 & 2.0 & 2.0 & 0.26230 \\
\hline 323.15 & 300 & 0.5 & 0.0 & 0.0 & 1.0 & 2.5 & 2.0 & 0.26013 \\
\hline 323.15 & 300 & 0.5 & 0.0 & 0.0 & 1.0 & 1.0 & 2.5 & 0.26220 \\
\hline 323.15 & 300 & 0.5 & 0.0 & 0.0 & 1.0 & 1.0 & 3.0 & 0.26229 \\
\hline 323.15 & 300 & 0.5 & 0.0 & 0.0 & 1.0 & 1.0 & 3.5 & 0.26073 \\
\hline 323.15 & 300 & 0.5 & 0.0 & 0.0 & 1.0 & 1.0 & 4.0 & 0.26227 \\
\hline 323.15 & 400 & 0.1 & 0.0 & 0.0 & 1.0 & 1.0 & 2.0 & 0.26801 \\
\hline 323.15 & 400 & 0.2 & 0.0 & 0.0 & 1.0 & 1.0 & 2.0 & 0.26516 \\
\hline 323.15 & 400 & 0.5 & 0.0 & 0.0 & 1.0 & 1.0 & 2.0 & 0.26226 \\
\hline 323.15 & 400 & 1.0 & 0.0 & 0.0 & 1.0 & 1.0 & 2.0 & 0.25593 \\
\hline 323.15 & 400 & 0.5 & 0.5 & 0.0 & 1.0 & 1.0 & 2.0 & 0.26013 \\
\hline 323.15 & 400 & 0.5 & 1.0 & 0.0 & 1.0 & 1.0 & 2.0 & 0.25374 \\
\hline 323.15 & 400 & 0.5 & 1.5 & 0.0 & 1.0 & 1.0 & 2.0 & 0.24961 \\
\hline 323.15 & 400 & 0.5 & 0.0 & 0.5 & 1.0 & 1.0 & 2.0 & 0.25582 \\
\hline 323.15 & 400 & 0.5 & 0.0 & 1.0 & 1.0 & 1.0 & 2.0 & 0.25330 \\
\hline 323.15 & 400 & 0.5 & 0.0 & 1.5 & 1.0 & 1.0 & 2.0 & 0.24941 \\
\hline 323.15 & 400 & 0.5 & 0.0 & 0.0 & 1.5 & 1.0 & 2.0 & 0.25732 \\
\hline 323.15 & 400 & 0.5 & 0.0 & 0.0 & 2.0 & 1.0 & 2.0 & 0.25279 \\
\hline 323.15 & 400 & 0.5 & 0.0 & 0.0 & 2.5 & 1.0 & 2.0 & 0.24859 \\
\hline 323.15 & 400 & 0.5 & 0.0 & 0.0 & 1.0 & 1.5 & 2.0 & 0.26013 \\
\hline
\end{tabular}




\begin{tabular}{|c|c|c|c|c|c|c|c|c|}
\hline 323.15 & 400 & 0.5 & 0.0 & 0.0 & 1.0 & 2.0 & 2.0 & 0.25731 \\
\hline 323.15 & 400 & 0.5 & 0.0 & 0.0 & 1.0 & 2.5 & 2.0 & 0.25524 \\
\hline 323.15 & 400 & 0.5 & 0.0 & 0.0 & 1.0 & 1.0 & 2.5 & 0.25721 \\
\hline 323.15 & 400 & 0.5 & 0.0 & 0.0 & 1.0 & 1.0 & 3.0 & 0.25730 \\
\hline 323.15 & 400 & 0.5 & 0.0 & 0.0 & 1.0 & 1.0 & 3.5 & 0.25589 \\
\hline 323.15 & 400 & 0.5 & 0.0 & 0.0 & 1.0 & 1.0 & 4.0 & 0.25731 \\
\hline 333.15 & 100 & 0.1 & 0.0 & 0.0 & 1.0 & 1.0 & 2.0 & 0.25333 \\
\hline 333.15 & 100 & 0.2 & 0.0 & 0.0 & 1.0 & 1.0 & 2.0 & 0.25055 \\
\hline 333.15 & 100 & 0.5 & 0.0 & 0.0 & 1.0 & 1.0 & 2.0 & 0.24786 \\
\hline 333.15 & 100 & 1.0 & 0.0 & 0.0 & 1.0 & 1.0 & 2.0 & 0.24178 \\
\hline 333.15 & 100 & 0.5 & 0.5 & 0.0 & 1.0 & 1.0 & 2.0 & 0.24592 \\
\hline 333.15 & 100 & 0.5 & 1.0 & 0.0 & 1.0 & 1.0 & 2.0 & 0.23983 \\
\hline 333.15 & 100 & 0.5 & 1.5 & 0.0 & 1.0 & 1.0 & 2.0 & 0.23588 \\
\hline 333.15 & 100 & 0.5 & 0.0 & 0.5 & 1.0 & 1.0 & 2.0 & 0.24178 \\
\hline 333.15 & 100 & 0.5 & 0.0 & 1.0 & 1.0 & 1.0 & 2.0 & 0.23938 \\
\hline 333.15 & 100 & 0.5 & 0.0 & 1.5 & 1.0 & 1.0 & 2.0 & 0.23571 \\
\hline 333.15 & 100 & 0.5 & 0.0 & 0.0 & 1.5 & 1.0 & 2.0 & 0.24311 \\
\hline 333.15 & 100 & 0.5 & 0.0 & 0.0 & 2.0 & 1.0 & 2.0 & 0.23885 \\
\hline 333.15 & 100 & 0.5 & 0.0 & 0.0 & 2.5 & 1.0 & 2.0 & 0.23496 \\
\hline 333.15 & 100 & 0.5 & 0.0 & 0.0 & 1.0 & 1.5 & 2.0 & 0.24583 \\
\hline 333.15 & 100 & 0.5 & 0.0 & 0.0 & 1.0 & 2.0 & 2.0 & 0.24328 \\
\hline 333.15 & 100 & 0.5 & 0.0 & 0.0 & 1.0 & 2.5 & 2.0 & 0.24122 \\
\hline 333.15 & 100 & 0.5 & 0.0 & 0.0 & 1.0 & 1.0 & 2.5 & 0.24308 \\
\hline 333.15 & 100 & 0.5 & 0.0 & 0.0 & 1.0 & 1.0 & 3.0 & 0.24316 \\
\hline 333.15 & 100 & 0.5 & 0.0 & 0.0 & 1.0 & 1.0 & 3.5 & 0.24172 \\
\hline 333.15 & 100 & 0.5 & 0.0 & 0.0 & 1.0 & 1.0 & 4.0 & 0.24316 \\
\hline 333.15 & 200 & 0.1 & 0.0 & 0.0 & 1.0 & 1.0 & 2.0 & 0.25077 \\
\hline 333.15 & 200 & 0.2 & 0.0 & 0.0 & 1.0 & 1.0 & 2.0 & 0.24803 \\
\hline 333.15 & 200 & 0.5 & 0.0 & 0.0 & 1.0 & 1.0 & 2.0 & 0.24539 \\
\hline 333.15 & 200 & 1.0 & 0.0 & 0.0 & 1.0 & 1.0 & 2.0 & 0.23937 \\
\hline 333.15 & 200 & 0.5 & 0.5 & 0.0 & 1.0 & 1.0 & 2.0 & 0.24339 \\
\hline 333.15 & 200 & 0.5 & 1.0 & 0.0 & 1.0 & 1.0 & 2.0 & 0.23754 \\
\hline 333.15 & 200 & 0.5 & 1.5 & 0.0 & 1.0 & 1.0 & 2.0 & 0.23359 \\
\hline 333.15 & 200 & 0.5 & 0.0 & 0.5 & 1.0 & 1.0 & 2.0 & 0.23942 \\
\hline 333.15 & 200 & 0.5 & 0.0 & 1.0 & 1.0 & 1.0 & 2.0 & 0.23699 \\
\hline 333.15 & 200 & 0.5 & 0.0 & 1.5 & 1.0 & 1.0 & 2.0 & 0.23340 \\
\hline 333.15 & 200 & 0.5 & 0.0 & 0.0 & 1.5 & 1.0 & 2.0 & 0.24069 \\
\hline 333.15 & 200 & 0.5 & 0.0 & 0.0 & 2.0 & 1.0 & 2.0 & 0.23660 \\
\hline 333.15 & 200 & 0.5 & 0.0 & 0.0 & 2.5 & 1.0 & 2.0 & 0.23263 \\
\hline 333.15 & 200 & 0.5 & 0.0 & 0.0 & 1.0 & 1.5 & 2.0 & 0.24337 \\
\hline 333.15 & 200 & 0.5 & 0.0 & 0.0 & 1.0 & 2.0 & 2.0 & 0.24078 \\
\hline 333.15 & 200 & 0.5 & 0.0 & 0.0 & 1.0 & 2.5 & 2.0 & 0.23890 \\
\hline 333.15 & 200 & 0.5 & 0.0 & 0.0 & 1.0 & 1.0 & 2.5 & 0.24067 \\
\hline 333.15 & 200 & 0.5 & 0.0 & 0.0 & 1.0 & 1.0 & 3.0 & 0.24079 \\
\hline
\end{tabular}




\begin{tabular}{|c|c|c|c|c|c|c|c|c|}
\hline 333.15 & 200 & 0.5 & 0.0 & 0.0 & 1.0 & 1.0 & 3.5 & 0.23937 \\
\hline 333.15 & 200 & 0.5 & 0.0 & 0.0 & 1.0 & 1.0 & 4.0 & 0.24073 \\
\hline 333.15 & 300 & 0.1 & 0.0 & 0.0 & 1.0 & 1.0 & 2.0 & 0.24229 \\
\hline 333.15 & 300 & 0.2 & 0.0 & 0.0 & 1.0 & 1.0 & 2.0 & 0.23978 \\
\hline 333.15 & 300 & 0.5 & 0.0 & 0.0 & 1.0 & 1.0 & 2.0 & 0.23711 \\
\hline 333.15 & 300 & 1.0 & 0.0 & 0.0 & 1.0 & 1.0 & 2.0 & 0.23143 \\
\hline 333.15 & 300 & 0.5 & 0.5 & 0.0 & 1.0 & 1.0 & 2.0 & 0.23524 \\
\hline 333.15 & 300 & 0.5 & 1.0 & 0.0 & 1.0 & 1.0 & 2.0 & 0.22951 \\
\hline 333.15 & 300 & 0.5 & 1.5 & 0.0 & 1.0 & 1.0 & 2.0 & 0.22586 \\
\hline 333.15 & 300 & 0.5 & 0.0 & 0.5 & 1.0 & 1.0 & 2.0 & 0.23141 \\
\hline 333.15 & 300 & 0.5 & 0.0 & 1.0 & 1.0 & 1.0 & 2.0 & 0.22907 \\
\hline 333.15 & 300 & 0.5 & 0.0 & 1.5 & 1.0 & 1.0 & 2.0 & 0.22564 \\
\hline 333.15 & 300 & 0.5 & 0.0 & 0.0 & 1.5 & 1.0 & 2.0 & 0.23271 \\
\hline 333.15 & 300 & 0.5 & 0.0 & 0.0 & 2.0 & 1.0 & 2.0 & 0.22863 \\
\hline 333.15 & 300 & 0.5 & 0.0 & 0.0 & 2.5 & 1.0 & 2.0 & 0.22499 \\
\hline 333.15 & 300 & 0.5 & 0.0 & 0.0 & 1.0 & 1.5 & 2.0 & 0.23523 \\
\hline 333.15 & 300 & 0.5 & 0.0 & 0.0 & 1.0 & 2.0 & 2.0 & 0.23270 \\
\hline 333.15 & 300 & 0.5 & 0.0 & 0.0 & 1.0 & 2.5 & 2.0 & 0.23085 \\
\hline 333.15 & 300 & 0.5 & 0.0 & 0.0 & 1.0 & 1.0 & 2.5 & 0.23271 \\
\hline 333.15 & 300 & 0.5 & 0.0 & 0.0 & 1.0 & 1.0 & 3.0 & 0.23267 \\
\hline 333.15 & 300 & 0.5 & 0.0 & 0.0 & 1.0 & 1.0 & 3.5 & 0.23139 \\
\hline 333.15 & 300 & 0.5 & 0.0 & 0.0 & 1.0 & 1.0 & 4.0 & 0.23271 \\
\hline 333.15 & 400 & 0.1 & 0.0 & 0.0 & 1.0 & 1.0 & 2.0 & 0.23771 \\
\hline 333.15 & 400 & 0.2 & 0.0 & 0.0 & 1.0 & 1.0 & 2.0 & 0.23519 \\
\hline 333.15 & 400 & 0.5 & 0.0 & 0.0 & 1.0 & 1.0 & 2.0 & 0.23273 \\
\hline 333.15 & 400 & 1.0 & 0.0 & 0.0 & 1.0 & 1.0 & 2.0 & 0.22710 \\
\hline 333.15 & 400 & 0.5 & 0.5 & 0.0 & 1.0 & 1.0 & 2.0 & 0.23080 \\
\hline 333.15 & 400 & 0.5 & 1.0 & 0.0 & 1.0 & 1.0 & 2.0 & 0.22527 \\
\hline 333.15 & 400 & 0.5 & 1.5 & 0.0 & 1.0 & 1.0 & 2.0 & 0.22172 \\
\hline 333.15 & 400 & 0.5 & 0.0 & 0.5 & 1.0 & 1.0 & 2.0 & 0.22706 \\
\hline 333.15 & 400 & 0.5 & 0.0 & 1.0 & 1.0 & 1.0 & 2.0 & 0.22487 \\
\hline 333.15 & 400 & 0.5 & 0.0 & 1.5 & 1.0 & 1.0 & 2.0 & 0.22148 \\
\hline 333.15 & 400 & 0.5 & 0.0 & 0.0 & 1.5 & 1.0 & 2.0 & 0.22828 \\
\hline 333.15 & 400 & 0.5 & 0.0 & 0.0 & 2.0 & 1.0 & 2.0 & 0.22443 \\
\hline 333.15 & 400 & 0.5 & 0.0 & 0.0 & 2.5 & 1.0 & 2.0 & 0.22076 \\
\hline 333.15 & 400 & 0.5 & 0.0 & 0.0 & 1.0 & 1.5 & 2.0 & 0.23080 \\
\hline 333.15 & 400 & 0.5 & 0.0 & 0.0 & 1.0 & 2.0 & 2.0 & 0.22838 \\
\hline 333.15 & 400 & 0.5 & 0.0 & 0.0 & 1.0 & 2.5 & 2.0 & 0.22663 \\
\hline 333.15 & 400 & 0.5 & 0.0 & 0.0 & 1.0 & 1.0 & 2.5 & 0.22830 \\
\hline 333.15 & 400 & 0.5 & 0.0 & 0.0 & 1.0 & 1.0 & 3.0 & 0.22844 \\
\hline 333.15 & 400 & 0.5 & 0.0 & 0.0 & 1.0 & 1.0 & 3.5 & 0.22705 \\
\hline 333.15 & 400 & 0.5 & 0.0 & 0.0 & 1.0 & 1.0 & 4.0 & 0.22833 \\
\hline 343.15 & 100 & 0.1 & 0.0 & 0.0 & 1.0 & 1.0 & 2.0 & 0.20363 \\
\hline 343.15 & 100 & 0.2 & 0.0 & 0.0 & 1.0 & 1.0 & 2.0 & 0.20162 \\
\hline
\end{tabular}




\begin{tabular}{|c|c|c|c|c|c|c|c|c|}
\hline 343.15 & 100 & 0.5 & 0.0 & 0.0 & 1.0 & 1.0 & 2.0 & 0.19947 \\
\hline 343.15 & 100 & 1.0 & 0.0 & 0.0 & 1.0 & 1.0 & 2.0 & 0.19488 \\
\hline 343.15 & 100 & 0.5 & 0.5 & 0.0 & 1.0 & 1.0 & 2.0 & 0.19793 \\
\hline 343.15 & 100 & 0.5 & 1.0 & 0.0 & 1.0 & 1.0 & 2.0 & 0.19338 \\
\hline 343.15 & 100 & 0.5 & 1.5 & 0.0 & 1.0 & 1.0 & 2.0 & 0.19048 \\
\hline 343.15 & 100 & 0.5 & 0.0 & 0.5 & 1.0 & 1.0 & 2.0 & 0.19488 \\
\hline 343.15 & 100 & 0.5 & 0.0 & 1.0 & 1.0 & 1.0 & 2.0 & 0.19305 \\
\hline 343.15 & 100 & 0.5 & 0.0 & 1.5 & 1.0 & 1.0 & 2.0 & 0.19025 \\
\hline 343.15 & 100 & 0.5 & 0.0 & 0.0 & 1.5 & 1.0 & 2.0 & 0.19588 \\
\hline 343.15 & 100 & 0.5 & 0.0 & 0.0 & 2.0 & 1.0 & 2.0 & 0.19266 \\
\hline 343.15 & 100 & 0.5 & 0.0 & 0.0 & 2.5 & 1.0 & 2.0 & 0.18975 \\
\hline 343.15 & 100 & 0.5 & 0.0 & 0.0 & 1.0 & 1.5 & 2.0 & 0.19796 \\
\hline 343.15 & 100 & 0.5 & 0.0 & 0.0 & 1.0 & 2.0 & 2.0 & 0.19592 \\
\hline 343.15 & 100 & 0.5 & 0.0 & 0.0 & 1.0 & 2.5 & 2.0 & 0.19443 \\
\hline 343.15 & 100 & 0.5 & 0.0 & 0.0 & 1.0 & 1.0 & 2.5 & 0.19590 \\
\hline 343.15 & 100 & 0.5 & 0.0 & 0.0 & 1.0 & 1.0 & 3.0 & 0.19590 \\
\hline 343.15 & 100 & 0.5 & 0.0 & 0.0 & 1.0 & 1.0 & 3.5 & 0.19488 \\
\hline 343.15 & 100 & 0.5 & 0.0 & 0.0 & 1.0 & 1.0 & 4.0 & 0.19594 \\
\hline 343.15 & 200 & 0.1 & 0.0 & 0.0 & 1.0 & 1.0 & 2.0 & 0.20168 \\
\hline 343.15 & 200 & 0.2 & 0.0 & 0.0 & 1.0 & 1.0 & 2.0 & 0.19964 \\
\hline 343.15 & 200 & 0.5 & 0.0 & 0.0 & 1.0 & 1.0 & 2.0 & 0.19769 \\
\hline 343.15 & 200 & 1.0 & 0.0 & 0.0 & 1.0 & 1.0 & 2.0 & 0.19305 \\
\hline 343.15 & 200 & 0.5 & 0.5 & 0.0 & 1.0 & 1.0 & 2.0 & 0.19618 \\
\hline 343.15 & 200 & 0.5 & 1.0 & 0.0 & 1.0 & 1.0 & 2.0 & 0.19159 \\
\hline 343.15 & 200 & 0.5 & 1.5 & 0.0 & 1.0 & 1.0 & 2.0 & 0.18861 \\
\hline 343.15 & 200 & 0.5 & 0.0 & 0.5 & 1.0 & 1.0 & 2.0 & 0.19305 \\
\hline 343.15 & 200 & 0.5 & 0.0 & 1.0 & 1.0 & 1.0 & 2.0 & 0.19130 \\
\hline 343.15 & 200 & 0.5 & 0.0 & 1.5 & 1.0 & 1.0 & 2.0 & 0.18846 \\
\hline 343.15 & 200 & 0.5 & 0.0 & 0.0 & 1.5 & 1.0 & 2.0 & 0.19405 \\
\hline 343.15 & 200 & 0.5 & 0.0 & 0.0 & 2.0 & 1.0 & 2.0 & 0.19084 \\
\hline 343.15 & 200 & 0.5 & 0.0 & 0.0 & 2.5 & 1.0 & 2.0 & 0.18790 \\
\hline 343.15 & 200 & 0.5 & 0.0 & 0.0 & 1.0 & 1.5 & 2.0 & 0.19618 \\
\hline 343.15 & 200 & 0.5 & 0.0 & 0.0 & 1.0 & 2.0 & 2.0 & 0.19411 \\
\hline 343.15 & 200 & 0.5 & 0.0 & 0.0 & 1.0 & 2.5 & 2.0 & 0.19265 \\
\hline 343.15 & 200 & 0.5 & 0.0 & 0.0 & 1.0 & 1.0 & 2.5 & 0.19404 \\
\hline 343.15 & 200 & 0.5 & 0.0 & 0.0 & 1.0 & 1.0 & 3.0 & 0.19409 \\
\hline 343.15 & 200 & 0.5 & 0.0 & 0.0 & 1.0 & 1.0 & 3.5 & 0.19302 \\
\hline 343.15 & 200 & 0.5 & 0.0 & 0.0 & 1.0 & 1.0 & 4.0 & 0.19416 \\
\hline 343.15 & 300 & 0.1 & 0.0 & 0.0 & 1.0 & 1.0 & 2.0 & 0.19526 \\
\hline 343.15 & 300 & 0.2 & 0.0 & 0.0 & 1.0 & 1.0 & 2.0 & 0.19337 \\
\hline 343.15 & 300 & 0.5 & 0.0 & 0.0 & 1.0 & 1.0 & 2.0 & 0.19134 \\
\hline 343.15 & 300 & 1.0 & 0.0 & 0.0 & 1.0 & 1.0 & 2.0 & 0.18694 \\
\hline 343.15 & 300 & 0.5 & 0.5 & 0.0 & 1.0 & 1.0 & 2.0 & 0.18988 \\
\hline 343.15 & 300 & 0.5 & 1.0 & 0.0 & 1.0 & 1.0 & 2.0 & 0.18563 \\
\hline
\end{tabular}




\begin{tabular}{|c|c|c|c|c|c|c|c|c|}
\hline 343.15 & 300 & 0.5 & 1.5 & 0.0 & 1.0 & 1.0 & 2.0 & 0.18270 \\
\hline 343.15 & 300 & 0.5 & 0.0 & 0.5 & 1.0 & 1.0 & 2.0 & 0.18697 \\
\hline 343.15 & 300 & 0.5 & 0.0 & 1.0 & 1.0 & 1.0 & 2.0 & 0.18519 \\
\hline 343.15 & 300 & 0.5 & 0.0 & 1.5 & 1.0 & 1.0 & 2.0 & 0.18259 \\
\hline 343.15 & 300 & 0.5 & 0.0 & 0.0 & 1.5 & 1.0 & 2.0 & 0.18800 \\
\hline 343.15 & 300 & 0.5 & 0.0 & 0.0 & 2.0 & 1.0 & 2.0 & 0.18494 \\
\hline 343.15 & 300 & 0.5 & 0.0 & 0.0 & 2.5 & 1.0 & 2.0 & 0.18202 \\
\hline 343.15 & 300 & 0.5 & 0.0 & 0.0 & 1.0 & 1.5 & 2.0 & 0.18997 \\
\hline 343.15 & 300 & 0.5 & 0.0 & 0.0 & 1.0 & 2.0 & 2.0 & 0.18798 \\
\hline 343.15 & 300 & 0.5 & 0.0 & 0.0 & 1.0 & 2.5 & 2.0 & 0.18653 \\
\hline 343.15 & 300 & 0.5 & 0.0 & 0.0 & 1.0 & 1.0 & 2.5 & 0.18791 \\
\hline 343.15 & 300 & 0.5 & 0.0 & 0.0 & 1.0 & 1.0 & 3.0 & 0.18803 \\
\hline 343.15 & 300 & 0.5 & 0.0 & 0.0 & 1.0 & 1.0 & 3.5 & 0.18691 \\
\hline 343.15 & 300 & 0.5 & 0.0 & 0.0 & 1.0 & 1.0 & 4.0 & 0.18796 \\
\hline 343.15 & 400 & 0.1 & 0.0 & 0.0 & 1.0 & 1.0 & 2.0 & 0.19176 \\
\hline 343.15 & 400 & 0.2 & 0.0 & 0.0 & 1.0 & 1.0 & 2.0 & 0.18986 \\
\hline 343.15 & 400 & 0.5 & 0.0 & 0.0 & 1.0 & 1.0 & 2.0 & 0.18804 \\
\hline 343.15 & 400 & 1.0 & 0.0 & 0.0 & 1.0 & 1.0 & 2.0 & 0.18368 \\
\hline 343.15 & 400 & 0.5 & 0.5 & 0.0 & 1.0 & 1.0 & 2.0 & 0.18661 \\
\hline 343.15 & 400 & 0.5 & 1.0 & 0.0 & 1.0 & 1.0 & 2.0 & 0.18228 \\
\hline 343.15 & 400 & 0.5 & 1.5 & 0.0 & 1.0 & 1.0 & 2.0 & 0.17955 \\
\hline 343.15 & 400 & 0.5 & 0.0 & 0.5 & 1.0 & 1.0 & 2.0 & 0.18366 \\
\hline 343.15 & 400 & 0.5 & 0.0 & 1.0 & 1.0 & 1.0 & 2.0 & 0.18197 \\
\hline 343.15 & 400 & 0.5 & 0.0 & 1.5 & 1.0 & 1.0 & 2.0 & 0.17950 \\
\hline 343.15 & 400 & 0.5 & 0.0 & 0.0 & 1.5 & 1.0 & 2.0 & 0.18469 \\
\hline 343.15 & 400 & 0.5 & 0.0 & 0.0 & 2.0 & 1.0 & 2.0 & 0.18163 \\
\hline 343.15 & 400 & 0.5 & 0.0 & 0.0 & 2.5 & 1.0 & 2.0 & 0.17895 \\
\hline 343.15 & 400 & 0.5 & 0.0 & 0.0 & 1.0 & 1.5 & 2.0 & 0.18654 \\
\hline 343.15 & 400 & 0.5 & 0.0 & 0.0 & 1.0 & 2.0 & 2.0 & 0.18465 \\
\hline 343.15 & 400 & 0.5 & 0.0 & 0.0 & 1.0 & 2.5 & 2.0 & 0.18328 \\
\hline 343.15 & 400 & 0.5 & 0.0 & 0.0 & 1.0 & 1.0 & 2.5 & 0.18469 \\
\hline 343.15 & 400 & 0.5 & 0.0 & 0.0 & 1.0 & 1.0 & 3.0 & 0.18463 \\
\hline 343.15 & 400 & 0.5 & 0.0 & 0.0 & 1.0 & 1.0 & 3.5 & 0.18366 \\
\hline 343.15 & 400 & 0.5 & 0.0 & 0.0 & 1.0 & 1.0 & 4.0 & 0.18463 \\
\hline 353.15 & 100 & 0.1 & 0.0 & 0.0 & 1.0 & 1.0 & 2.0 & 0.16604 \\
\hline 353.15 & 100 & 0.2 & 0.0 & 0.0 & 1.0 & 1.0 & 2.0 & 0.16451 \\
\hline 353.15 & 100 & 0.5 & 0.0 & 0.0 & 1.0 & 1.0 & 2.0 & 0.16282 \\
\hline 353.15 & 100 & 1.0 & 0.0 & 0.0 & 1.0 & 1.0 & 2.0 & 0.15932 \\
\hline 353.15 & 100 & 0.5 & 0.5 & 0.0 & 1.0 & 1.0 & 2.0 & 0.16160 \\
\hline 353.15 & 100 & 0.5 & 1.0 & 0.0 & 1.0 & 1.0 & 2.0 & 0.15809 \\
\hline 353.15 & 100 & 0.5 & 1.5 & 0.0 & 1.0 & 1.0 & 2.0 & 0.15574 \\
\hline 353.15 & 100 & 0.5 & 0.0 & 0.5 & 1.0 & 1.0 & 2.0 & 0.15924 \\
\hline 353.15 & 100 & 0.5 & 0.0 & 1.0 & 1.0 & 1.0 & 2.0 & 0.15789 \\
\hline 353.15 & 100 & 0.5 & 0.0 & 1.5 & 1.0 & 1.0 & 2.0 & 0.15573 \\
\hline
\end{tabular}




\begin{tabular}{|c|c|c|c|c|c|c|c|c|}
\hline 353.15 & 100 & 0.5 & 0.0 & 0.0 & 1.5 & 1.0 & 2.0 & 0.16001 \\
\hline 353.15 & 100 & 0.5 & 0.0 & 0.0 & 2.0 & 1.0 & 2.0 & 0.15760 \\
\hline 353.15 & 100 & 0.5 & 0.0 & 0.0 & 2.5 & 1.0 & 2.0 & 0.15517 \\
\hline 353.15 & 100 & 0.5 & 0.0 & 0.0 & 1.0 & 1.5 & 2.0 & 0.16163 \\
\hline 353.15 & 100 & 0.5 & 0.0 & 0.0 & 1.0 & 2.0 & 2.0 & 0.16004 \\
\hline 353.15 & 100 & 0.5 & 0.0 & 0.0 & 1.0 & 2.5 & 2.0 & 0.15890 \\
\hline 353.15 & 100 & 0.5 & 0.0 & 0.0 & 1.0 & 1.0 & 2.5 & 0.16010 \\
\hline 353.15 & 100 & 0.5 & 0.0 & 0.0 & 1.0 & 1.0 & 3.0 & 0.16013 \\
\hline 353.15 & 100 & 0.5 & 0.0 & 0.0 & 1.0 & 1.0 & 3.5 & 0.15921 \\
\hline 353.15 & 100 & 0.5 & 0.0 & 0.0 & 1.0 & 1.0 & 4.0 & 0.16014 \\
\hline 353.15 & 200 & 0.1 & 0.0 & 0.0 & 1.0 & 1.0 & 2.0 & 0.16455 \\
\hline 353.15 & 200 & 0.2 & 0.0 & 0.0 & 1.0 & 1.0 & 2.0 & 0.16292 \\
\hline 353.15 & 200 & 0.5 & 0.0 & 0.0 & 1.0 & 1.0 & 2.0 & 0.16136 \\
\hline 353.15 & 200 & 1.0 & 0.0 & 0.0 & 1.0 & 1.0 & 2.0 & 0.15790 \\
\hline 353.15 & 200 & 0.5 & 0.5 & 0.0 & 1.0 & 1.0 & 2.0 & 0.16016 \\
\hline 353.15 & 200 & 0.5 & 1.0 & 0.0 & 1.0 & 1.0 & 2.0 & 0.15669 \\
\hline 353.15 & 200 & 0.5 & 1.5 & 0.0 & 1.0 & 1.0 & 2.0 & 0.15437 \\
\hline 353.15 & 200 & 0.5 & 0.0 & 0.5 & 1.0 & 1.0 & 2.0 & 0.15782 \\
\hline 353.15 & 200 & 0.5 & 0.0 & 1.0 & 1.0 & 1.0 & 2.0 & 0.15649 \\
\hline 353.15 & 200 & 0.5 & 0.0 & 1.5 & 1.0 & 1.0 & 2.0 & 0.15428 \\
\hline 353.15 & 200 & 0.5 & 0.0 & 0.0 & 1.5 & 1.0 & 2.0 & 0.15867 \\
\hline 353.15 & 200 & 0.5 & 0.0 & 0.0 & 2.0 & 1.0 & 2.0 & 0.15610 \\
\hline 353.15 & 200 & 0.5 & 0.0 & 0.0 & 2.5 & 1.0 & 2.0 & 0.15383 \\
\hline 353.15 & 200 & 0.5 & 0.0 & 0.0 & 1.0 & 1.5 & 2.0 & 0.16026 \\
\hline 353.15 & 200 & 0.5 & 0.0 & 0.0 & 1.0 & 2.0 & 2.0 & 0.15864 \\
\hline 353.15 & 200 & 0.5 & 0.0 & 0.0 & 1.0 & 2.5 & 2.0 & 0.15758 \\
\hline 353.15 & 200 & 0.5 & 0.0 & 0.0 & 1.0 & 1.0 & 2.5 & 0.15860 \\
\hline 353.15 & 200 & 0.5 & 0.0 & 0.0 & 1.0 & 1.0 & 3.0 & 0.15860 \\
\hline 353.15 & 200 & 0.5 & 0.0 & 0.0 & 1.0 & 1.0 & 3.5 & 0.15780 \\
\hline 353.15 & 200 & 0.5 & 0.0 & 0.0 & 1.0 & 1.0 & 4.0 & 0.15870 \\
\hline 353.15 & 300 & 0.1 & 0.0 & 0.0 & 1.0 & 1.0 & 2.0 & 0.15952 \\
\hline 353.15 & 300 & 0.2 & 0.0 & 0.0 & 1.0 & 1.0 & 2.0 & 0.15801 \\
\hline 353.15 & 300 & 0.5 & 0.0 & 0.0 & 1.0 & 1.0 & 2.0 & 0.15647 \\
\hline 353.15 & 300 & 1.0 & 0.0 & 0.0 & 1.0 & 1.0 & 2.0 & 0.15309 \\
\hline 353.15 & 300 & 0.5 & 0.5 & 0.0 & 1.0 & 1.0 & 2.0 & 0.15545 \\
\hline 353.15 & 300 & 0.5 & 1.0 & 0.0 & 1.0 & 1.0 & 2.0 & 0.15200 \\
\hline 353.15 & 300 & 0.5 & 1.5 & 0.0 & 1.0 & 1.0 & 2.0 & 0.14988 \\
\hline 353.15 & 300 & 0.5 & 0.0 & 0.5 & 1.0 & 1.0 & 2.0 & 0.15307 \\
\hline 353.15 & 300 & 0.5 & 0.0 & 1.0 & 1.0 & 1.0 & 2.0 & 0.15174 \\
\hline 353.15 & 300 & 0.5 & 0.0 & 1.5 & 1.0 & 1.0 & 2.0 & 0.14968 \\
\hline 353.15 & 300 & 0.5 & 0.0 & 0.0 & 1.5 & 1.0 & 2.0 & 0.15383 \\
\hline 353.15 & 300 & 0.5 & 0.0 & 0.0 & 2.0 & 1.0 & 2.0 & 0.15156 \\
\hline 353.15 & 300 & 0.5 & 0.0 & 0.0 & 2.5 & 1.0 & 2.0 & 0.14934 \\
\hline 353.15 & 300 & 0.5 & 0.0 & 0.0 & 1.0 & 1.5 & 2.0 & 0.15536 \\
\hline
\end{tabular}




\begin{tabular}{lllllllll}
353.15 & 300 & 0.5 & 0.0 & 0.0 & 1.0 & 2.0 & 2.0 & 0.15396 \\
353.15 & 300 & 0.5 & 0.0 & 0.0 & 1.0 & 2.5 & 2.0 & 0.15275 \\
353.15 & 300 & 0.5 & 0.0 & 0.0 & 1.0 & 1.0 & 2.5 & 0.15384 \\
353.15 & 300 & 0.5 & 0.0 & 0.0 & 1.0 & 1.0 & 3.0 & 0.15384 \\
353.15 & 300 & 0.5 & 0.0 & 0.0 & 1.0 & 1.0 & 3.5 & 0.15307 \\
353.15 & 300 & 0.5 & 0.0 & 0.0 & 1.0 & 1.0 & 4.0 & 0.15395 \\
353.15 & 400 & 0.1 & 0.0 & 0.0 & 1.0 & 1.0 & 2.0 & 0.15692 \\
353.15 & 400 & 0.2 & 0.0 & 0.0 & 1.0 & 1.0 & 2.0 & 0.15534 \\
353.15 & 400 & 0.5 & 0.0 & 0.0 & 1.0 & 1.0 & 2.0 & 0.15395 \\
353.15 & 400 & 1.0 & 0.0 & 0.0 & 1.0 & 1.0 & 2.0 & 0.15056 \\
353.15 & 400 & 0.5 & 0.5 & 0.0 & 1.0 & 1.0 & 2.0 & 0.15274 \\
353.15 & 400 & 0.5 & 1.0 & 0.0 & 1.0 & 1.0 & 2.0 & 0.14948 \\
353.15 & 400 & 0.5 & 1.5 & 0.0 & 1.0 & 1.0 & 2.0 & 0.14741 \\
353.15 & 400 & 0.5 & 0.0 & 0.5 & 1.0 & 1.0 & 2.0 & 0.15053 \\
353.15 & 400 & 0.5 & 0.0 & 1.0 & 1.0 & 1.0 & 2.0 & 0.14930 \\
353.15 & 400 & 0.5 & 0.0 & 1.5 & 1.0 & 1.0 & 2.0 & 0.14721 \\
353.15 & 400 & 0.5 & 0.0 & 0.0 & 1.5 & 1.0 & 2.0 & 0.15128 \\
353.15 & 400 & 0.5 & 0.0 & 0.0 & 2.0 & 1.0 & 2.0 & 0.14904 \\
353.15 & 400 & 0.5 & 0.0 & 0.0 & 2.5 & 1.0 & 2.0 & 0.14680 \\
353.15 & 400 & 0.5 & 0.0 & 0.0 & 1.0 & 1.5 & 2.0 & 0.15285 \\
353.15 & 400 & 0.5 & 0.0 & 0.0 & 1.0 & 2.0 & 2.0 & 0.15133 \\
353.15 & 400 & 0.5 & 0.0 & 0.0 & 1.0 & 2.5 & 2.0 & 0.15022 \\
353.15 & 400 & 0.5 & 0.0 & 0.0 & 1.0 & 1.0 & 2.5 & 0.15127 \\
353.15 & 400 & 0.5 & 0.0 & 0.0 & 1.0 & 1.0 & 3.0 & 0.15138 \\
353.15 & 400 & 0.5 & 0.0 & 0.0 & 1.0 & 1.0 & 3.5 & 0.15051 \\
353.15 & 400 & 0.5 & 0.0 & 0.0 & 1.0 & 1.0 & 4.0 & 0.15131 \\
\hline & & & & & & & &
\end{tabular}

Table S2 Measured supersolubility of $\mathrm{Li}_{2} \mathrm{CO}_{3}$ (Orthogonal experiments)

\begin{tabular}{ccccccccc}
\hline $\begin{array}{c}\text { Temperature } \\
(\mathrm{K})\end{array}$ & $\begin{array}{c}\text { agitation } \\
\text { speed } \\
(\mathrm{rpm})\end{array}$ & $\begin{array}{c}\text { Volume } \\
(\mathrm{L})\end{array}$ & $\begin{array}{c}\mathrm{c}\left(\mathrm{K}_{2} \mathrm{SO}_{4}\right) \\
(\mathrm{mol} / \mathrm{L})\end{array}$ & $\begin{array}{c}\mathrm{c}(\mathrm{NaCl}) \\
(\mathrm{mol} / \mathrm{L})\end{array}$ & $\begin{array}{c}\mathrm{c}\left(\mathrm{Li}_{2} \mathrm{SO}_{4}\right) \\
(\mathrm{mol} / \mathrm{L})\end{array}$ & $\begin{array}{c}\mathrm{c}\left(\mathrm{Na}_{2} \mathrm{CO}_{3}\right) \\
(\mathrm{mol} / \mathrm{L})\end{array}$ & $\begin{array}{c}\mathrm{Na}_{2} \mathrm{CO}_{3} \\
\text { feed rate } \\
(\mathrm{ml} / \mathrm{min})\end{array}$ & $\begin{array}{c}c_{s s} \\
(\mathrm{~mol} / \mathrm{L})\end{array}$ \\
\hline 313.15 & 100 & 0.1 & 0 & 0 & 1.0 & 1.0 & 2.0 & 0.33118 \\
313.15 & 150 & 0.3 & 0.9 & 1.2 & 2.0 & 2.2 & 2.3 & 0.27759 \\
313.15 & 200 & 0.6 & 1.8 & 0.3 & 1.6 & 2.0 & 2.6 & 0.26786 \\
313.15 & 250 & 1.0 & 0.6 & 1.5 & 1.2 & 1.8 & 2.9 & 0.26496 \\
313.15 & 300 & 0.2 & 1.5 & 0.6 & 2.2 & 1.6 & 3.2 & 0.26814 \\
313.15 & 350 & 0.5 & 0.3 & 1.8 & 1.8 & 1.4 & 3.5 & 0.26775 \\
313.15 & 400 & 0.8 & 1.2 & 0.9 & 1.4 & 1.2 & 3.8 & 0.25840 \\
319.15 & 100 & 1.0 & 1.5 & 1.2 & 1.6 & 1.4 & 3.8 & 0.22880 \\
319.15 & 150 & 0.2 & 0.3 & 0.3 & 1.2 & 1.2 & 2.0 & 0.28857 \\
319.15 & 200 & 0.5 & 1.2 & 1.5 & 2.2 & 1.0 & 2.3 & 0.24681 \\
319.15 & 250 & 0.8 & 0 & 0.6 & 1.8 & 2.2 & 2.6 & 0.25743 \\
319.15 & 300 & 0.1 & 0.9 & 1.8 & 1.4 & 2.0 & 2.9 & 0.25360
\end{tabular}




\begin{tabular}{|c|c|c|c|c|c|c|c|c|}
\hline 319.15 & 350 & 0.3 & 1.8 & 0.9 & 1.0 & 1.8 & 3.2 & 0.24714 \\
\hline 319.15 & 400 & 0.6 & 0.6 & 0 & 2.0 & 1.6 & 3.5 & 0.25101 \\
\hline 325.15 & 100 & 0.8 & 0.9 & 0.3 & 2.2 & 1.8 & 3.5 & 0.22033 \\
\hline 325.15 & 150 & 0.1 & 1.8 & 1.5 & 1.8 & 1.6 & 3.8 & 0.21718 \\
\hline 325.15 & 200 & 0.3 & 0.6 & 0.6 & 1.4 & 1.4 & 2.0 & 0.24991 \\
\hline 325.15 & 250 & 0.6 & 1.5 & 1.8 & 1.0 & 1.2 & 2.3 & 0.22562 \\
\hline 325.15 & 300 & 1.0 & 0.3 & 0.9 & 2.0 & 1.0 & 2.6 & 0.22720 \\
\hline 325.15 & 350 & 0.2 & 1.2 & 0 & 1.6 & 2.2 & 2.9 & 0.23585 \\
\hline 325.15 & 400 & 0.5 & 0 & 1.2 & 1.2 & 2.0 & 3.2 & 0.23550 \\
\hline 331.15 & 100 & 0.6 & 0.3 & 1.5 & 1.4 & 2.2 & 3.2 & 0.20644 \\
\hline 331.15 & 150 & 1.0 & 1.2 & 0.6 & 1.0 & 2.0 & 3.5 & 0.19764 \\
\hline 331.15 & 200 & 0.2 & 0 & 1.8 & 2.0 & 1.8 & 3.8 & 0.20642 \\
\hline 331.15 & 250 & 0.5 & 0.9 & 0.9 & 1.6 & 1.6 & 2.0 & 0.21299 \\
\hline 331.15 & 300 & 0.8 & 1.8 & 0 & 1.2 & 1.4 & 2.3 & 0.20558 \\
\hline 331.15 & 350 & 0.1 & 0.6 & 1.2 & 2.2 & 1.2 & 2.6 & 0.21298 \\
\hline 331.15 & 400 & 0.3 & 1.5 & 0.3 & 1.8 & 1.0 & 2.9 & 0.20778 \\
\hline 337.15 & 100 & 0.5 & 1.8 & 0.6 & 2.0 & 1.2 & 2.9 & 0.17853 \\
\hline 337.15 & 150 & 0.8 & 0.6 & 1.8 & 1.6 & 1.0 & 3.2 & 0.17843 \\
\hline 337.15 & 200 & 0.1 & 1.5 & 0.9 & 1.2 & 2.2 & 3.5 & 0.18333 \\
\hline 337.15 & 250 & 0.3 & 0.3 & 0 & 2.2 & 2.0 & 3.8 & 0.18754 \\
\hline 337.15 & 300 & 0.6 & 1.2 & 1.2 & 1.8 & 1.8 & 2.0 & 0.18134 \\
\hline 337.15 & 350 & 1.0 & 0 & 0.3 & 1.4 & 1.6 & 2.3 & 0.19148 \\
\hline 337.15 & 400 & 0.2 & 0.9 & 1.5 & 1.0 & 1.4 & 2.6 & 0.19015 \\
\hline 343.15 & 100 & 0.3 & 1.2 & 1.8 & 1.2 & 1.6 & 2.6 & 0.16221 \\
\hline 343.15 & 150 & 0.6 & 0 & 0.9 & 2.2 & 1.4 & 2.9 & 0.16480 \\
\hline 343.15 & 200 & 1.0 & 0.9 & 0 & 1.8 & 1.2 & 3.2 & 0.15812 \\
\hline 343.15 & 250 & 0.2 & 1.8 & 1.2 & 1.4 & 1.0 & 3.5 & 0.15727 \\
\hline 343.15 & 300 & 0.5 & 0.6 & 0.3 & 1.0 & 2.2 & 3.8 & 0.16345 \\
\hline 343.15 & 350 & 0.8 & 1.5 & 1.5 & 2.0 & 2.0 & 2.0 & 0.15143 \\
\hline 343.15 & 400 & 0.1 & 0.3 & 0.6 & 1.6 & 1.8 & 2.3 & 0.17406 \\
\hline 349.15 & 100 & 0.2 & 0.6 & 0.9 & 1.8 & 2.0 & 2.3 & 0.14536 \\
\hline 349.15 & 150 & 0.5 & 1.5 & 0 & 1.4 & 1.8 & 2.6 & 0.14098 \\
\hline 349.15 & 200 & 0.8 & 0.3 & 1.2 & 1.0 & 1.6 & 2.9 & 0.14075 \\
\hline 349.15 & 250 & 0.1 & 1.2 & 0.3 & 2.0 & 1.4 & 3.2 & 0.14112 \\
\hline 349.15 & 300 & 0.3 & 0 & 1.5 & 1.6 & 1.2 & 3.5 & 0.14183 \\
\hline 349.15 & 350 & 0.6 & 0.9 & 0.6 & 1.2 & 1.0 & 3.8 & 0.13758 \\
\hline 349.15 & 400 & 1.0 & 1.8 & 1.8 & 2.2 & 2.2 & 2.0 & 0.12475 \\
\hline
\end{tabular}

